CONSEJO DE ESTADO 



\title{
COMENTARIO AL DICTAMEN DEL CONSEJO DE ESTADO DE 1 DE MARZO DE 2012, SOBRE EL «ANTEPROYECTO DE LEY ORGÁNICA DE ESTABILIDAD PRESUPUESTARIA Y SOSTENIBILIDAD FINANCIERA»*
}

\author{
ISABEL M. GIMÉNEZ SÁNCHEZ
}

Profesora Titular Interina de Derecho Constitucional

Universidad Autónoma de Madrid

\begin{abstract}
SUMARIO
I. Introducción

II. Observaciones generales de carácter formal

III. Observaciones relativas al sistema de fuentes

IV. Observaciones referidas a asuntos sustantivos de la norma
\end{abstract}

\section{INTRODUCCIÓN}

La Comisión Permanente del Consejo de Estado, en sesión celebrada el día 1 de marzo de 2012, emitió, por unanimidad, un dictamen relativo al anteproyecto de Ley orgánica de Estabilidad Presupuestaria y Sostenibilidad Financiera de las Administraciones Públicas aprobado por el Consejo de Ministros el 13 de febrero. El objeto del presente comentario serán las principales cuestiones jurídicas abordadas por el citado Dictamen: Dada la necesaria brevedad de este formato, vaya por delante que no se pretende pasar revista a todos los aspectos señalados de forma pormenorizada en el Informe, sino simplemente y de manera forzosamente modesta, destacar aquellas cuestiones generales (y son bastante numerosas) que la Máxima autoridad consultiva pone de relieve en su Dictamen.

* Este estudio se ha realizado en el marco del Proyecto de Investigación del Ministerio de Ciencia e Innovación «Las reformas de los sistemas descentralizados». Ref. DER 2009-14235 cofinanciado con fondos FEDER. 
El informe fue solicitado por el Ministerio de Hacienda y Administraciones Públicas (Referencia: 164/2012) con fecha de 21 de febrero de 2012 (con registro de entrada el mismo día), a pesar de que, en un primer momento, el 27 de enero de 2012 se había anunciado la aprobación del mencionado anteproyecto, sin previsión de someterlo al preceptivo dictamen del Consejo de Estado. No obstante, como bien se señala en el propio Dictamen que ahora se comenta, la preceptividad del mismo trae causa de lo establecido en el número 2 del art. 21 de la Ley orgánica 3/1980, de 22 de abril, del Consejo de Estado (LOCE), según el cual «el Consejo de Estado en Pleno deberá ser consultado en los siguientes asuntos: (...) 2. Anteproyectos de leyes que hayan de dictarse en ejecución, cumplimiento o desarrollo de tratados, convenios o acuerdos internacionales y del derecho comunitario europeo». Asimismo, es de señalar que, por acuerdo del Consejo de Ministros de 17 de febrero de 2012 (adjunto al resto del expediente), se solicitaba que el dictamen fuera emitido en el plazo de nueve días naturales, por lo que, de conformidad con lo dispuesto en el número 2 del art. 19 LOCE, la consulta debió ser despachada por la Comisión Permanente ${ }^{1}$.

Por lo que respecta al texto del Dictamen, el Consejo de Estado comienza exponiendo de manera resumida el contenido del anteproyecto, así como del expediente que lo acompaña; se justifica tanto la competencia del propio órgano consultivo como la urgencia en la tramitación del informe, en los términos antes expuestos; y se da cuenta de algunas deficiencias observadas en la tramitación del proyecto, a las que más adelante me referiré. Pero sin duda, el aspecto más relevante de esta primera parte del Dictamen, antes de entrar a valorar las cuestiones de análisis del texto emanado del Consejo de Estado, es el completísimo estudio del marco normativo europeo de la estabilidad presupuestaria (desde sus orígenes en el Pacto de Estabilidad y Crecimiento de 1997 hasta el Tratado de Estabilidad, Coordinación y Gobernanza firmado el 2 de marzo de 2012, es decir, un día después de la aprobación del Dictamen objeto de este comentario), así como del marco normativo español en esta misma materia (desde las Leyes de 2001 hasta la reciente reforma constitucional operada en el art. $135 \mathrm{CE}$ ). De esta interesante exposición del marco normativo tanto europeo como nacional sobre la cuestión de la estabilidad presupuestaria, nada habré de añadir, sin embargo, más allá de destacar el interés de su lectura ${ }^{2}$.

Antes de entrar en el comentario del contenido del Dictamen, debo adelantar que no se seguirá aquí, debido a razones de mera claridad expositiva, la estructura seguida por el propio Consejo de Estado en su elaboración, esto es, mediante la disección capítulo a capítulo del anteproyecto, sino que he preferido sistematizar su estudio a partir de las distintas cuestiones tanto de índole formal como de contenido sustancial tratadas en el mis-

1 Así, se destacaba en el citado Acuerdo del Consejo de Ministros: «La importancia de las medidas de disciplina fiscal incorporadas en el anteproyecto, ante la gravedad de la situación económica, aconsejan adoptar cuanto antes las medidas previstas en el anteproyecto de Ley para garantizar la sostenibilidad financiera de todas las Administraciones Públicas, fortalecer la confianza en la estabilidad de la economía española y reforzar el compromiso de España con la Unión Europea en materia de estabilidad presupuestaria».

2 Para un mayor desarrollo de esta cuestión, que da cumplida cuenta de hasta qué punto la regulación contenida en el anteproyecto examinado trae su origen de las recientes reformas implementadas y exigidas en la Unión Europea, resultan imprescindible las lecturas de A. J. MENÉNDEZ, De la crisis económica a la crisis constitucional de la Unión Europea, Eolas, León, 2012, para el ámbito europeo; y A. EMBID IRUJO, La constitucionalización de la crisis económica, Iustel, 2012, para su recepción constitucional. 
mo. Así, en primer lugar analizaremos las cuestiones formales y generales, para después detenernos en los más relevantes aspectos sustantivos abordados en el Dictamen. Procuraré, asimismo, destacar aquellos aspectos del anteproyecto que hayan sido finalmente modificados (no demasiados, en realidad) en la redacción final acogida por la Ley Orgánica 2/2012, de 27 de abril, de Estabilidad Presupuestaria y Sostenibilidad Financiera (en adelante, LOEPSF), especialmente aquellos en los que se hayan acogido las propuestas contenidas en el Dictamen.

\section{OBSERVACIONES GENERALES DE TIPO FORMAL}

A este respecto, el Consejo de Estado señala algunas cuestiones de alcance general y sentido diverso. En primer lugar, y como observación previa, denuncia el carácter excesivamente breve y meramente descriptivo de la memoria anexa al Proyecto. Algo especialmente censurable en un tema tan complejo y técnico como es este, de modo que al no adjuntarse una explicación razonada y detallada de los distintos aspectos regulados, la tarea del Consejo de Estado se ha visto notablemente dificultada.

Otra cuestión de alcance general es la relativa al propio título del anteproyecto, ya que el Consejo de Estado considera preferible no incluir referencias al ámbito subjetivo de la norma, dado que precisamente este es un tema susceptible de generar confusión, puesto que según el Consejo, desde una perspectiva sustantiva, no refleja con exactitud dicho ámbito. Como se verá más adelante, uno de los principales problemas que planteaba el texto es el de la recepción de categorías procedentes del Derecho Europeo, que no siempre guardan una correspondencia con las existentes en el Derecho administrativo español.

Pero si hay una crítica que se reitere a lo largo del Dictamen en distintos pasajes y con la que no se puede menos que concordar es la relativa a la redacción del texto, tanto desde una perspectiva semántica como gramatical. El alcance de la crítica en este punto es de tal profundidad que llega a afirmar que «se estima necesaria una revisión del anteproyecto en su conjunto para mejorar la puntuación y corrección gramatical de algunas de sus previsiones». Lo mismo se reitera respecto de la necesidad de comprobar la corrección de las remisiones internas. Por lo que respecta a la terminología empleada, el Informe critica el abuso de expresiones traducidas literalmente del inglés, que en castellano resultan incorrectas (resulta llamativo el uso frecuente del adjetivo «neto» para indicar exclusión, o de la construcción «en términos de») o, simplemente, no se sabe muy bien a qué se refieren, como la famosa «senda hacia» el crecimiento económico o el equilibrio o de reducción de los desequilibrios... que insistentemente se repite a lo largo del anteproyecto y ha seguido tal cual en el texto definitivo de la LOEPSF.

De otra parte, el Consejo de Estado señala la conveniencia de iniciar la Exposición de Motivos «con una referencia expresa a la de la reforma constitucional, para subrayar el valor de la estabilidad presupuestaria desde la perspectiva del artículo 135 de la Constitución». Esta observación se repite en otros pasajes del Dictamen, en los que el Consejo de Estado insiste en la necesidad de recordar en el texto del anteproyecto que la aprobación de esta ley trae causa de la remisión explícita que la reciente reforma del art. 135 CE hace a una ley orgánica para desarrollar los principios de estabilidad presupuestaria y sos- 
tenibilidad financiera según lo enunciado en el propio precepto constitucional. Esta observación resulta de especial interés por cuanto es cierto que el borrador presentado por el Gobierno elude resaltar este vínculo directo con la Constitución, mientras que constantemente se referencia a la normativa y a las exigencias europeas, lo cual, sin dejar de responder a la realidad político-económica, no resulta jurídicamente adecuado. Asimismo, tal y como se analizará más adelante, no cabe olvidar que el título competencial que habilita al legislador a regular esta materia, con la intensidad y el alcance que lo hace, no se encuentra (pese a lo que en un primer momento indicaba el anteproyecto) en el art. $149 \mathrm{CE}$, sino precisamente en el mencionado art. $135 \mathrm{CE}$, tras la reciente reforma constitucional llevada a cabo en el segundo semestre de 2011.

Por último, el Consejo de Estado echa en falta una referencia precisa y más detallada a las disposiciones europeas que se encuentran en el origen del texto proyectado. También en este sentido, una de las principales novedades que introduce este anteproyecto es que el ámbito de aplicación subjetivo de la Ley proyectada pasa a definirse con arreglo al Sistema Europeo de Cuentas Nacionales y Regionales, que es el que toma como base la normativa europea sobre estabilidad presupuestaria.

\section{OBSERVACIONES REFERIDAS A ASPECTOS RELATIVOS AL SISTEMA DE FUENTES}

\section{LEGISLACIÓN BÁSICA}

El apartado 2 de la disposición final primera del anteproyecto establecía como base competencial los arts. $149.1 .13^{\mathrm{a}}$ y $149.1 .18^{\mathrm{a}} \mathrm{CE}$, al tiempo que atribuía a todo su contenido el carácter de legislación básica del Estado. Frente a esta previsión, el Consejo de Estado considera que la atribución de carácter básico a la totalidad de los preceptos del anteproyecto, precisamente al amparo de los títulos competenciales de los arts. 149.1.13 y $18^{\mathrm{a}}$ de la Constitución, resulta incoherente por un lado, con la invocación en la disposición final primera- de las competencias estatales exclusivas y excluyentes sobre el «sistema monetario» y la «Hacienda general y Deuda del Estado» del art.

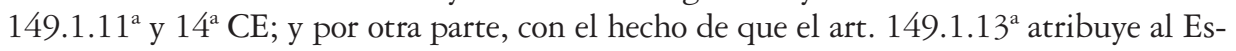
tado competencia exclusiva no solo sobre las «bases» sino también sobre la «coordinación» de la planificación general de la actividad económica, que por su propia naturaleza no puede considerarse como meramente básica. A lo cual habría de añadirse que, del análisis de conjunto del anteproyecto, se desprende que el contenido de la regulación de la generalidad de los preceptos no admite desarrollo normativo alguno por parte de las Comunidades Autónomas, lo cual es el rasgo característico de la normativa básica ${ }^{3}$.

En el fondo, como muy directamente pone de relieve el Dictamen, «el carácter básico que se otorga al anteproyecto obedece al deseo de garantizar que sus previsiones re-

3 «[A] sí, resulta difícil pensar en qué medida podrían las Comunidades Autónomas desarrollar — por ejemplo- el límite de deuda pública establecido en el artículo 13.1, las medidas de cumplimiento forzoso previstas en el artículo 26 o los mecanismos de control de constitucionalidad contemplados en la disposición adicional tercera» 
sulten de aplicación a todas las Administraciones públicas». Pero dado que el anteproyecto trae origen directamente de la «remisión a una Ley orgánica, obviamente estatal» establecida en el art. $135 \mathrm{CE}$, «carece del carácter de legislación básica y, en consecuencia, debería ser desarrollado por el ejecutivo estatal, salvo cuando se haga una remisión expresa al desarrollo autonómico (por ejemplo, artículo 31, párrafo segundo) o se trate de materias que, por su propia naturaleza, admitan dicho desarrollo (por ejemplo, medidas preventivas - artículo 18- o transparencia —artículo 27-), en cuyo caso deberán preservar lo establecido por la Ley orgánica proyectada, sin que para ello sea necesario que su contenido haya sido calificado de básico».

Todo ello, por tanto, deriva nuevamente de la cuestión anteriormente considerada por el Consejo de Estado, relativa a la conveniencia de mencionar, como único fundamento competencial del anteproyecto, el art. $135 \mathrm{CE}$, suprimiendo tanto la referencia a los títulos competenciales del art. 149.1 CE, como al carácter básico de la norma.

\section{Contenido de la Ley Orgánica}

Trayendo causa de lo anterior, respecto de que el art. 135 CE remite a una ley orgánica posterior el desarrollo del principio de estabilidad presupuestaria, el anteproyecto sometido a consulta se encuadra en el supuesto del último inciso del art. $81 \mathrm{CE}$. El Consejo de Estado considera que esta remisión supone la creación de «una suerte de reserva de ley orgánica específica», que implicaría que «el desarrollo del citado precepto constitucional, en los aspectos en él mencionados, únicamente puede verificarse, en este primer escalón normativo, a través de ese preciso instrumento normativo».

Recuerda, asimismo, el Consejo de Estado que «la relación ley orgánica-ley no orgánica se articula básicamente con arreglo al principio de competencia, que opera a partir del acotamiento y consiguiente separación de ámbitos competenciales diferentes, cuyo tratamiento se reserva a órganos y procedimientos determinados con exclusión de todos los demás posibles». En este sentido, es claro que el nuevo art. 135 CE acota expresamente un ámbito competencial preciso, por referencia a los principios de estabilidad presupuestaria y de sostenibilidad financiera, que se concretan respectivamente en los objetivos de déficit y de volumen de deuda, así como al principio de responsabilidad. Considera el Consejo que no se da en cambio esa conexión explícita en el caso de los principios de plurianualidad, de transparencia, de lealtad institucional y de eficiencia en la asignación de los recursos públicos, si bien admite su posible justificación debido a que «constituyen complementos necesarios para garantizar un mejor y más eficaz cumplimiento de los aludidos objetivos de deuda y déficit».

Por otra parte, partiendo del presupuesto de que el mencionado art. $135 \mathrm{CE}$ defiere a una ley orgánica el desarrollo de los principios en él contenidos, así como la regulación de la participación, en los procedimientos respectivos, de los órganos de coordinación institucional entre las Administraciones Públicas en materia de política fiscal y financiera, el Consejo de Estado pasa a analizar el contenido concreto del anteproyecto, con el fin de comprobar si cumple de manera satisfactoria con el encargo de regulación normativa que la reserva de ley orgánica realizada por la Constitución conlleva, de manera tal que no puede entenderse admisible una remisión en blanco al Gobierno, para su 
ulterior desarrollo reglamentario. Asimismo, se destaca que en el art. 135 CE, así como en el apartado 2 de la disposición adicional que acompaña la reforma (que remite a la ley orgánica el establecimiento de los mecanismos que permitan el cumplimiento del límite de deuda a que se refiere el propio artículo 135.3 del texto constitucional) se mencionan expresamente algunos aspectos de dicho desarrollo que necesariamente por tanto deberán regularse en la futura Ley orgánica.

Tras realizar un repaso de la regulación contenida en el anteproyecto, el Consejo de Estado concluye que «en líneas generales y sin perjuicio de las concretas observaciones que puedan formularse más adelante, que la Ley orgánica proyectada aborda de modo suficiente el desarrollo del principio de estabilidad presupuestaria». No obstante, desde esta perspectiva general de adecuación a las habilitaciones contenidas en el texto constitucional, el Consejo formula dos observaciones de interés.

En primer lugar, la participación de los órganos de coordinación institucional — tanto el Consejo de Política Fiscal y Financiera de las Comunidades Autónomas (CPFF) como la Comisión Nacional de Administración Local (CNAL) - se contempla principalmente al hilo de los distintos procedimientos regulados en el anteproyecto, bien mediante la exigencia de su informe previo o bien por el requisito de que el órgano estatal correspondiente les suministre determinada información. Considera, sin embargo, el Consejo de Estado que, para dar adecuado cumplimiento del mandato constitucional, sería necesaria una previsión que recogiera desde una perspectiva más general, el «conjunto de las funciones que a dichos órganos pueden, si no deben, corresponder en el ámbito de la estabilidad presupuestaria».

Otra cuestión que se plantea en este contexto el Consejo de Estado es la de si la demora en la entrada en vigor hasta el año 2020, que la Constitución únicamente prevé para los límites de déficit estructural, puede o no extenderse mediante la LOEPSF también al límite de deuda. Según afirma el Dictamen comentado, resulta evidente de la disposición transitoria primera del anteproyecto, que en la actualidad no se cumple la exigencia de que el volumen de deuda pública del conjunto de Administraciones Públicas no supere el 60 por ciento del Producto Interior Bruto nacional expresado en términos nominales. En este sentido, el Máximo órgano consultivo emplea un razonamiento eminentemente racional y realista para concluir que, dada la imposibilidad de exigir su cumplimiento en las actuales circunstancias, «parece lógico que la vigencia del límite de deuda tenga el mismo horizonte temporal que el de gasto».

\section{Control de COnstitucionalidad}

En este punto el Dictamen recuerda con todo detalle la diversidad de mecanismos de control de constitucionalidad establecidos en el art. $161 \mathrm{CE}$ y desarrollados en la LOTC en función de su objeto y de las diferentes consecuencias que estos conllevan, centrándose especialmente en el estudio de la «singularidad del procedimiento impugnatorio contemplado en el artículo 161.2 de la Constitución y el título V de la LOTC, respecto del recurso de inconstitucionalidad y del conflicto (positivo) de competencia». En realidad, el problema venía dado por el hecho de que el anteproyecto sólo mencionase al Gobierno, como órgano facultado para impugnar ante el Tribunal Constitucional tales 
leyes, disposiciones o actos, lo cual podía suscitar dudas en el caso de leyes, disposiciones normativas o actos «con fuerza de ley» de las Comunidades Autónomas, donde la legitimación para interponer recurso de inconstitucionalidad viene atribuida por el art. 32.1 LOTC al Presidente del Gobierno, al Defensor del Pueblo, a cincuenta diputados o a cincuenta senadores ${ }^{4}$. En este caso el Consejo de Estado considera que si bien el "propósito del anteproyecto no es, desde luego, restringir los sujetos legitimados para la interposición del recurso de inconstitucionalidad cuando éste se plantee en materia de estabilidad presupuestaria», sería conveniente suprimir esa referencia al Gobierno, para evitar eventuales problemas interpretativos. «Por lo demás, debería decirse que los procedimientos de impugnación serán — como es obvio— los establecidos en la LOTC».

Pero más allá de este aspecto, si se quiere formal, la verdadera cuestión aquí estribaba en «los motivos que — según el anteproyecto- pueden justificar la activación de los mecanismos de control de constitucionalidad», al permitirse impugnar ante el Tribunal Constitucional las leyes y las disposiciones normativas o actos, con o sin fuerza de Ley, de las Comunidades Autónomas que «vulneren los principios establecidos en esta Ley». Y lo más relevante a este respecto, en opinión del Consejo de Estado, es que la ausencia en este precepto de toda referencia a los principios consagrados en el art. 135 de la Constitución «no parece fortuita». El motivo en el que se basa el Consejo para sostener esta afirmación es la ausencia de un criterio definido acerca del papel que las jurisdicciones constitucional y contencioso-administrativa debían tener en la fiscalización de los leyes, disposiciones normativas o actos, con o sin fuerza de ley — sobre todo estos-, que fuesen contrarios a la LOEPSF durante la tramitación del expediente 5 .

En estos supuestos, señala el Consejo de Estado, no cabe afirmar en abstracto que la vulneración de cualesquiera principios o previsiones establecidos en una ley, ni siquiera cuando esta sea aprobada en desarrollo de un precepto constitucional, constituya per se un vicio de inconstitucionalidad. La distinción entre vicios de inconstitucionalidad e infracciones de mera legalidad, por tanto, debe hacerse inevitablemente atendiendo a las circunstancias del caso, por lo que «[u]na previsión de tal naturaleza difuminaría los límites que deben existir entre la jurisdicción constitucional y la contencioso-administrativa». Teniendo en cuenta lo anterior y, puesto que las infracciones de mera legalidad en ningún caso pueden ser conocidas por el Tribunal Constitucional, debería decirse que dicha impugnación sólo resultará procedente cuando las leyes, disposiciones o actos autonómicos «vulneren los principios establecidos en el artículo 135 de la Constitución y desarrollados en la presente Ley».

4 A diferencia de lo que ocurre con las disposiciones normativas, resoluciones o actos «sin fuerza de ley» de las Comunidades Autónomas, en los que la legitimación para plantear un conflicto positivo de competencia o acudir al procedimiento específico de impugnación del título $\mathrm{V}$ de la Constitución corresponde únicamente al Gobierno.

5 Indica el Consejo de Estado que «la disposición adicional segunda del texto inicial del anteproyecto, de fecha 26 de enero de 2012, preveía la competencia de la jurisdicción contencioso-administrativa para conocer de los recursos contra los actos y resoluciones dictados en aplicación de esta Ley orgánica; esta previsión ha sido sustituida por la actual disposición adicional tercera del anteproyecto sometido a consulta, en la que se admite la posibilidad de impugnación ante el Tribunal Constitucional de leyes, disposiciones normativas y actos, con o sin fuerza de ley, que «vulneren los principios establecidos en esta Ley». 


\section{Recurso contra una Ley de Presupuestos autonómica}

Esta cuestión viene referida a la previsión contenida en el apartado 2 de la disposición adicional tercera del anteproyecto, que ante una eventual impugnación de una ley de Presupuestos (de una Comunidad Autónoma) que produjera la suspensión de su vigencia, preveía la prórroga automática de los Presupuestos del ejercicio anterior «hasta la aprobación de los Presupuestos del ejercicio siguiente al impugnado o, en su caso, hasta el levantamiento de la suspensión de la ley impugnada». A este respecto, el Consejo de Estado comienza recordando la necesaria conexión de este precepto con el art. 161.2 CE y la doctrina jurisprudencial recaída en torno al mismo, recordando que la suspensión de las Leyes de Presupuestos recurridas sólo podrá producirse a iniciativa del Gobierno y su duración no podrá exceder de cinco meses, excepto acuerdo expreso del Tribunal Constitucional. Por este motivo, considera inexcusable la cita del referido art. 161.2 CE en el apartado 2 de la disposición adicional tercera del anteproyecto.

Por otra parte, reconoce el Consejo de Estado que sin duda la suspensión de las Leyes de Presupuestos autonómicas «incide en una de las facultades esenciales de la autonomía financiera de las Comunidades Autónomas reconocida en el artículo 156.1 de la Constitución, como es la de aprobar sus propios presupuestos». Sin embargo, como bien señala el Informe, la autonomía financiera de las Comunidades Autónomas —y la facultad que éstas tienen de aprobar sus propios presupuestosencuentra un límite en el principio de estabilidad presupuestaria, establecido en el art. $135.6 \mathrm{CE}$.

En relación con la cuestión de la prórroga automática de los Presupuestos del ejercicio anterior hasta el levantamiento de la suspensión o, si esto no sucede, hasta la aprobación de los Presupuestos del ejercicio siguiente al impugnado, en principio, para el Consejo de Estado «parece razonable que la suspensión de la Ley de Presupuestos recurrida traiga consigo la prórroga automática de los Presupuestos del ejercicio anterior durante el período de suspensión», dadas sus similitudes con la previsión del art. 134.4 CE para el supuesto de no aprobación de la Ley de Presupuestos. Cuestión distinta es si la suspensión de la Ley de Presupuestos recurrida y la consiguiente prórroga automática de los Presupuestos del ejercicio anterior, determinan que la Comunidad Autónoma no pueda aprobar una nueva Ley de Presupuestos hasta el ejercicio económico siguiente al impugnado, salvo que el levantamiento de la suspensión se produzca antes de la finalización de éste. En este punto la jurisprudencia constitucional no resulta del todo clara, aunque no parece evidente que los efectos de la suspensión puedan extenderse a la facultad de aprobar una nueva ley. Por este motivo, considera el Consejo de Estado que la excesiva simplicidad de la formulación del mencionado precepto del anteproyecto «no agota las diversas hipótesis que pudieran plantearse durante el ejercicio autonómico a resultas de ulteriores iniciativas legislativas autonómicas sobre la misma materia o materias conexas en los términos que acaban de ser vistos», ni tampoco resulta adecuada en aquellos supuestos — seguramente más probables- en los que la suspensión afectase sólo a determinados aspectos de la Ley impugnada. 
Estas observaciones — que tenían carácter esencial, con cualificación especial por cuanto podían lesionar el ejercicio de potestades legislativas, fueron finalmente acogidas por el legislador orgánico, dando cabida a los distintos supuestos planteados por el Consejo de Estado.

\section{OBSERVACIONES REFERIDAS A ASPECTOS SUSTANTIVOS DE LA NORMA}

El Consejo de Estado, en su Informe, lleva a cabo un repaso por las principales cuestiones que se introducen en el anteproyecto, que supone «una revisión en profundidad de la legislación vigente».

\section{RESPECTO DE LAS NOVEDADES INTRODUCIDAS EN LOS PRINCIPIOS GENERALES}

En relación con los principios generales, el anteproyecto sometido a consideración viene a modificar dos ya existentes: por un lado, en la nueva definición del principio de estabilidad presupuestaria se excluye cualquier referencia expresa al ciclo económico y, por otra parte, se amplía el alcance del principio de transparencia. Asimismo, se introducen tres nuevos principios: el de sostenibilidad financiera — que forma parte del título del anteproyecto y de la consiguiente LOEPSF_-, entendida como «la capacidad para financiar compromisos de gasto presentes y futuros dentro de los límites de déficit y deuda pública»; el de responsabilidad, que implica que aquella «Administración incumplidora o que contribuya a producir el incumplimiento de los compromisos asumidos por España de acuerdo con la normativa europea, asumirá, en la parte que le sea imputable, las responsabilidades que de tal incumplimiento se hubieran derivado»; y, por último, el de lealtad institucional, que engloba una serie de reglas que deberán ser respetadas por las Administraciones Públicas en sus actuaciones, «con la finalidad de armonizar y facilitar la colaboración y cooperación entre ellas en materia presupuestaria».

Pero, sin duda, los aspectos más importantes y novedosos son los relativos a la instrumentación de los dos principios que constituyen el núcleo del anteproyecto, esto es, los principios de estabilidad presupuestaria y sostenibilidad financiera. A este respecto el principio de estabilidad presupuestaria se traduce en «la exigencia de que todas las Administraciones Públicas deben presentar equilibrio o superávit, sin que puedan incurrir en déficit estructural, entendido como déficit ajustado del ciclo, sin tener en cuenta las medidas excepcionales y temporales». Se admiten, no obstante, dos flexibilizaciones: que en caso de reformas estructurales con efectos presupuestarios a largo plazo pueda alcanzarse en el conjunto de Administraciones Públicas un déficit estructural del 0,4\% del PIB; y que en aquellas situaciones excepcionales enumeradas en la LOEPSF (catástrofes naturales, recesión económica o situación de emergencia extraordinaria), que deberán ser apreciadas por la mayoría absoluta del Congreso de los Diputados, tanto el Estado como las Comunidades Autónomas podrán presentar déficit 
estructural. Por último, se incorpora la regla de gasto prevista en el Reglamento (UE) $\mathrm{n}^{\circ}$ 1175/2011, la célebre «regla de oro», según la cual el gasto de las Administraciones Públicas no podrá aumentar por encima de la tasa de crecimiento de referencia del Producto Interior Bruto.

En lo que concierne al principio de sostenibilidad financiera, este viene a traducirse en la exigencia de que «el volumen de deuda pública del conjunto de Administraciones Públicas no supere el $60 \%$ del PIB o el porcentaje que al efecto establezcan las normas europeas». Las circunstancias de flexibilización son las mismas que las previstas en relación al principio de estabilidad presupuestaria.

Siguiendo lo previsto en la reforma del art. $135 \mathrm{CE}$, se establece que los créditos presupuestarios para satisfacer los intereses y el capital de la deuda pública de las Administraciones se entenderán siempre incluidos en el estado de gastos de sus presupuestos, así como la prioridad absoluta de pago de los intereses y el capital de la deuda pública frente a cualquier otro tipo de gasto. Asimismo, se determinan los criterios para el establecimiento de los objetivos de estabilidad presupuestaria y de deuda pública para el conjunto de Administraciones Públicas. En el caso de las Comunidades Autónomas, se prevé la formulación de una propuesta para cada una de ellas, al tiempo que se determinan los informes sobre cumplimiento de los objetivos en cada uno de los principios antes mencionados, de cara a una eventual actuación preventiva y, llegado el caso, de ejecución de medidas correctivas.

A juicio del Consejo suscitan dudas las previsiones del anteproyecto relativas a la metodología y procedimiento para el cálculo del déficit estructural en cuanto a su adecuación al mandato constitucional. Esto es así dado que, en realidad, en el texto proyectado únicamente se afirma que «para el cálculo del déficit estructural se seguirá la metodología utilizada por la Comisión Europea en el marco de aplicación de la normativa de estabilidad presupuestaria» (apartado 6 del art. 11); y la disposición transitoria segunda se limita a señalar que «el Ministerio de Economía y Competitividad desarrollará la aplicación de la metodología prevista en el apartado 6 del artículo 11 sobre el cálculo del déficit estructural (...) al marco nacional en los tres meses siguientes a la aprobación de esta Ley». Según el Consejo de Estado, el anteproyecto no da adecuado cumplimiento al mandato contenido en el artículo 135.5.b) de la Constitución y, por tanto, la regulación proyectada sugiere completarla, como mínimo mencionando expresamente las normas europeas en que se ampara la competencia de la Comisión. Asimismo, sugiere incorporar al anteproyecto, al menos, las líneas generales de esa metodología, en lo que se refiere a los términos en que se remite al desarrollo reglamentario la aplicación de dicha metodología al marco nacional, pareciendo razonable que sea un Real Decreto el que aborde en un primer nivel reglamentario dicha regulación. Por último, defiende la necesidad de introducir alguna previsión «sobre el procedimiento a seguir para el cálculo del déficit estructural, cuestión respecto de la que el anteproyecto guarda silencio».

\section{RESPECTO DE LA ARTICULACIÓN DE MEDIDAS PREVENTIVAS, CORRECTIVAS Y COERCITIVAS}


a) Medidas preventivas:

En lo que respecta a las medidas preventivas, se prevé un mecanismo automático de prevención para garantizar que no se incurre en déficit estructural al final de cada ejercicio, junto con un umbral de deuda de carácter preventivo para evitar la superación de los límites establecidos. No obstante, a juicio del Consejo de Estado resulta necesaria una aclaración en cuanto a la delimitación del supuesto de hecho que determina la entrada en juego de la medida automática aquí prevista, por lo que sugiere la conveniencia de aclararlo.

Paralelamente, se instrumenta un procedimiento de «alerta temprana», de manera tal que la formulación de una advertencia permitirá que se anticipen las medidas necesarias de corrección en caso de que se aprecie riesgo de incumplimiento de los objetivos de estabilidad, de deuda pública o de la regla de gasto; de no adoptarse tales medidas se procederá a la aplicación de las medidas correctivas. Esta advertencia de riesgo de incumplimiento no es ya una medida automática, sino que requiere una previa apreciación del riesgo por parte del Gobierno. A este respecto, señala el Consejo de Estado que ese «riesgo de incumplimiento» que legitima la formulación de la advertencia «constituye un concepto jurídico indeterminado de no poca amplitud y cuya apreciación se atribuye al Gobierno que, en su caso, formulará una advertencia a la Administración responsable» y reconoce la dificultad de precisarlo, dada la imposibilidad de definir a priori las circunstancias que pueden provocar la existencia del riesgo de incumplimiento. No obstante, dada la trascendencia de las consecuencias que pueden derivar de dicha advertencia, se hace preciso «adoptar ciertas cautelas» y considera «imprescindible la exigencia de que la advertencia sea motivada, y que se trate de una motivación detallada que permita a la Administración responsable, en su caso, justificar la inexistencia de riesgo o argumentar su discrepancia con la advertencia formulada y de tan relevantes consecuencias». A este requisito de motivación de la decisión, el Consejo de Estado añade la necesidad de que con carácter previo a la formulación de la advertencia, se oiga a la Administración que va a ser advertida.

También desde el punto de vista procedimental, el anteproyecto preveía la necesidad de dar cuenta de la formulación de la advertencia, para su conocimiento, al Consejo de Política Fiscal y Financiera (si la advertida es una Comunidad Autónoma), o a la Comisión Nacional de Administración Local (si se trata de una Corporación Local). Como bien señala el Consejo de Estado, la intervención de los mencionados órganos es ciertamente limitada en este caso, puesto que se trata de una mera comunicación de la advertencia ya formulada, sin que puedan expresar parecer u opinión al respecto. Considera el Consejo que «esta intervención tan limitada de los órganos de coordinación y cooperación sería coherente con unas medidas netamente preventivas que supusieran un escaso grado de intervención estatal», pero dado que la formulación de la advertencia acarrea consecuencias de mayor intensidad (también desde la perspectiva del principio de autonomía), ello justificaría conceder una mayor trascendencia a la participación de los órganos de cooperación y coordinación mencionados. Considera el Consejo que la LOEPSF ha de conseguir el equilibrio entre la necesidad de rapidez de actuación exigidas por la efectividad de los principios recogidos en el art. $135 \mathrm{CE}$, por un lado, y las garantías derivadas del principio de autonomía, por otro. Por ello, sugiere el Consejo de Estado que tal participación se anteponga a la formulación de la advertencia, de manera que el Gobierno, antes de for- 
mular la advertencia, oiga al Consejo de Política Fiscal y Financiera o a la Comisión Nacional de la Administración Local, lo que le permitirá tener en cuenta el punto de vista de las demás Comunidades Autónomas o Corporaciones Locales.

Por otra parte, el Consejo de Estado advierte del riesgo de que el anteproyecto añada que la advertencia «se hará pública para general conocimiento», lo cual puede acarrear serias consecuencias políticas y económicas. Por ello, el Consejo de Estado sugiere introducir «una referencia expresa al principio de lealtad institucional, incluso con alguna especificación que garantice su efectividad en este punto».

En cuanto a las consecuencias jurídicas que derivan de la advertencia una vez formulada, se establecía el plazo de un mes para adoptar las medidas necesarias para evitar el riesgo; y, en caso de que éstas no se adoptaran o el Ministro de Hacienda y Administraciones Públicas apreciase que son insuficientes para conjurar el riesgo, «se aplicarán las medidas correctivas previstas en los artículos 20 y $21 »$. Esto plantea un problema, según señala el Consejo de Estado, en cuanto a que las medidas correctivas se aplicarían «cuando todavía no se ha producido el incumplimiento, esto es, cuando existe sólo una situación de riesgo — no actualizado-, apreciado por el citado órgano estatal (quizás en discrepancia con la Administración afectada)». Por ello, el Consejo de Estado propone introducir «un nivel intermedio de medidas, preventivas aún y no ya correctivas, específicas en función del concreto riesgo de que se trate; podría tratarse incluso de una previsión de medidas ad hoc, de menor intensidad que las medidas correctivas». Y ello, recuerda una vez más el Consejo de Estado, en un intento de preservar el principio de autonomía.

b) Medidas correctivas.

Respecto de las medidas correctivas, se establecen, en primer lugar, medidas automáticas de corrección, consistentes en la exigencia de obtener una autorización previa para las emisiones de deuda, la concesión de subvenciones o la celebración de convenios. Por otra parte, el incumplimiento de alguno de los objetivos — de estabilidad presupuestaria, de deuda pública o de la regla de gastos - genera la obligación de presentar un plan económico-financiero que permita la corrección de la desviación en el plazo de un año, excepto en aquellos supuestos en los que se trate de un supuesto de déficit originado por circunstancias excepcionales, para los que se establece la obligación de presentar un plan de reequilibrio.

Empezando por las llamadas «medidas automáticas», puntualiza el Consejo de Estado que en realidad constituyen medidas que parecen derivar de la apreciación, por parte del Gobierno, de una situación de incumplimiento, al igual que ocurría con las anteriormente mencionadas medidas previas de advertencia de riesgo. Sin embargo, el Consejo de Estado denuncia la falta de claridad del supuesto de hecho, cuando se habla de «los supuestos de incumplimiento», sin aclarar ni el órgano que ha de apreciarlo ni los criterios que ha de seguir para hacerlo, por lo que considera que es necesaria una previsión dirigida a establecer, con carácter general, cuándo ha de considerarse producido el incumplimiento, cómo ha de producirse tal constatación y a quién corresponde apreciarla y, en consecuencia, poder así determinar el preciso momento en que son de aplicación las medidas correctivas.

La principal medida correctiva recogida en el Anteproyecto, consiste en la formulación de un plan económico-financiero, que el Consejo de Estado considera una exi- 
gencia admisible desde la perspectiva constitucional, con base en la reciente jurisprudencia constitucional. En cuanto a la concreta regulación prevista, de nuevo se plantean problemas de falta de precisión en el supuesto de hecho, por lo que el Consejo de Estado advierte de la necesidad de una previsión destinada a establecer, con carácter general, cuándo ha de considerarse producido el incumplimiento y en qué términos debe producirse tal declaración. Y lo mismo respecto de la consecuencia jurídica, anudada a dicho incumplimiento, puesto que ni se establece el órgano responsable de la formulación del plan, ni cuál debe ser el objetivo final del mismo, entre otras varias cuestiones abiertas. Otro tanto puede decirse sobre las observaciones realizadas por el Consejo de Estado respecto de la siguiente medida correctiva: el plan de reequilibrio. También aquí se critica la falta de precisión del texto y se conmina a determinar a quién corresponde la elaboración y aprobación de los planes de reequilibrio, en aquellos supuestos en los que los límites de deuda pública superados sean los de las Corporaciones Locales.

Respecto de la tramitación y seguimiento de los planes y, más en concreto, de los plazos para su aprobación, el Consejo de Estado hace notar las dificultades que podrían seguir de la formulación empleada por el anteproyecto, que dispone que los mismos serán «elaborados» en el plazo máximo de un mes «desde que se constate el incumplimiento» o se aprecien las circunstancias previstas en el artículo 11.3. El Consejo considera preferible comenzar el cómputo no desde su elaboración (que es un proceso) sino desde su aprobación (que cierra aquel proceso), especialmente teniendo en cuenta que en algunos supuestos la elaboración corresponderá al Gobierno, mientras la aprobación recaerá sobre las Cortes Generales, con el consiguiente lapso temporal entre ambos.

Pero sin duda los principales reparos los plantea el Consejo de Estado en relación con la intervención de Consejo de Política Fiscal y Financiera (CPFF), ya que los planes elaborados por las Comunidades Autónomas serán remitidos al CPFF, que comprobará la idoneidad de las medidas incluidas y la adecuación de sus previsiones a los objetivos fijados. Si este órgano considera que las medidas contenidas en el plan presentado no garantizan la corrección de la situación de desequilibrio, requerirá a la Comunidad Autónoma en cuestión la presentación de un nuevo plan; pero si la Comunidad Autónoma no presenta el nuevo plan en plazo, o las medidas contenidas en el mismo no son suficientes a juicio del CPFF, «se aplicarán las medidas coercitivas previstas en el artículo 25».

Frente a las dudas referidas a una presunta vulneración del principio de autonomía financiera de las Comunidades Autónomas, respecto de un papel decisivo del CPFF que podría, incluso, dar lugar a la aplicación de las medidas coercitivas, el Consejo de Estado pasa revista a la más reciente jurisprudencia constitucional, haciendo especial hincapié en la nuclear STC 134/2011, de 20 de julio. Según dicha jurisprudencia, entre las competencias estatales figura de forma explícita la coordinación en diversos preceptos de la Constitución, con el alcance previsto en cada uno de ellos; y, en estos casos en que existe una atribución constitucional expresa, el alcance de los acuerdos de los órganos coordinadores será el que se derive del ejercicio de la correspondiente competencia. Y respecto del concreto papel del CPFF, recuerda el Consejo de Estado que la técnica del requerimiento a la Comunidad Autónoma ha sido considerada legítima por el Tribunal Constitucional, dado que consiste en comunicar la anomalía al órgano competente a fin de que éste remedie el incumplimiento detectado, por lo que no es sino un instrumento que permite al Estado velar para que no se produzcan diferencias en la ejecución o aplicación del 
bloque normativo, reconociendo la legitimidad de una función de vigilancia del Estado sobre las actuaciones ejecutivas de las Comunidades Autónomas.

A la vista de la doctrina expuesta, entiende el Consejo de Estado que la regulación recogida en el artículo 23.3 del anteproyecto puede considerarse conforme con la Constitución, si bien «el anteproyecto recoge una vía intermedia entre el simple reenvío (que podría ser indefinido) a la Comunidad Autónoma y la sustitución de la competencia autonómica, al imponer, tras una primera devolución, la aplicación de las medidas coercitivas del artículo 25». Dichas medidas son susceptibles, por tanto, de afectar al principio de autonomía. Sin embargo, en la citada STC 134/2011 se admite la posibilidad de establecer límites a la autonomía financiera de las Comunidades Autónomas, «derivados del principio de coordinación y de las funciones de coordinación que al Estado corresponden en materia económica y financiera. A ello cabe añadir ahora que la nueva redacción del artículo 135 de la Constitución (aprobado por la reforma de 27 de diciembre de 2011) introduce una regulación que entraña la aparición de nuevos límites a la autonomía financiera de las Comunidades Autónomas, al imponer determinadas exigencias y restricciones a «todas las Administraciones públicas»». En definitiva, entiende el Consejo de Estado que en sí mismo no vulnera la Constitución «el establecimiento de un respaldo sancionador (configurado en este caso como «medidas coercitivas») que garantice, en última instancia, la corrección de las desviaciones que se produzcan sobre los límites de déficit y de deuda, habida cuenta de que la propia Constitución impone, de forma expresa, la corrección de esas desviaciones». Otra cosa será, como el propio Consejo matiza, la admisibilidad de las concretas medidas, su alcance y sus límites.

Una especial mención dedica el Consejo de Estado al papel del CPFF respecto de los informes de seguimiento. A diferencia de lo que ocurre respecto de los informes de seguimiento de los planes, en este caso, el elemento desencadenante del requerimiento primero, y de la aplicación de las medidas coercitivas después, es un informe elaborado por el Ministro de Hacienda y Administraciones Públicas y no la postura del CPFF, que es «el órgano que traduce o en el que se refleja el marco constitucional de coordinación y cooperación entre el Estado y las Comunidades Autónomas en materia financiera, por lo que parece razonable atribuirle la apreciación de las circunstancias que pueden desembocar en la aplicación de las medidas coercitivas previstas en la norma proyectada», lo cual parece desprenderse también de la propia lectura del art. 135 CE, por lo que, una vez más, el Consejo de Estado entiende que en este mecanismo de corrección «debería darse una participación mucho más relevante al Consejo de Política Fiscal y Financiera (y, en su caso, de la Comisión Nacional de la Administración Local) en la adopción de las decisiones o en la constatación de las circunstancias que pueden determinar la aplicación de las medidas coercitivas del artículo 25 proyectado», si bien entiende que «ello debe entenderse sin perjuicio de que pueda atribuirse el poder último de decisión al Gobierno cuando esté en juego el cumplimiento de las normas de la Unión Europea y para garantizar su efectivo cumplimiento».

Por lo que respecta, en definitiva, a estas medidas correctivas, establecidas en el anteproyecto, en términos generales el Consejo de Estado reprocha que no se haya precisado el papel del CFFF. Se nos antoja un reproche bastante débil, puesto que no se trata sólo de falta de definición de este órgano, sino que a lo largo de todo el Dictamen, el Consejo de Estado parece dar a entender, aunque tampoco lo manifiesta con total rotundidad que, en aras del respeto al principio de autonomía, las diferentes decisiones no 
deberían ser adoptadas de manera unilateral por el Gobierno central. En realidad, estos procedimientos introducidos en el anteproyecto, recuerdan en muchos aspectos a la aplicación del procedimiento por déficit excesivo introducido en la Unión Europea por el artículo 104 del Tratado CE, como instrumento clave para garantizar la consecución de los objetivos del Pacto de Estabilidad y Crecimiento. Este procedimiento por déficit excesivo, ya planteó graves problemas institucionales en el ámbito institucional europeo, pero no cabe olvidarse que allí intervenían la Comisión y más tarde el Consejo ${ }^{6}$, mientras que en el nuevo procedimiento que ahora propone el Anteproyecto (y continúa en la redacción definitiva de la LOEPSP) la decisión de iniciar los diversos procedimientos depende únicamente del Ministerio de Hacienda.

c) Medidas coercitivas y de ejecución forzosa:-

Finalmente, en el anteproyecto se establecen medidas coercitivas y de ejecución forzosa. Dentro de las primeras, frente al supuesto de incumplimiento de un plan económico-financiero, se establece que la Administración responsable aprobará la no disponibilidad de créditos para garantizar el cumplimiento del objetivo establecido, con la obligación de constituir un depósito hasta entonces (depósito que se transformará en multa coercitiva en caso de persistir el incumplimiento durante seis meses). Al cabo de tres meses, corresponderá a una delegación de expertos la propuesta de las medidas que considere oportunas.

En último término, las medidas de ejecución forzosa únicamente están previstas para aquellos supuestos de no adopción de los acuerdos de no disponibilidad de créditos ni de las medidas propuestas por la delegación de expertos. Estas medidas extremas, para el caso en que la Administración incumplidora sea una Comunidad Autónoma, consisten en la incorporación del mecanismo previsto en el art. $155 \mathrm{CE}$, esto es, un requerimiento a su Presidente y, de no ser atendido, adopción de las medidas necesarias para obligar a la Comunidad Autónoma al cumplimiento forzoso. Para el supuesto, en cambio, de que incumplan las Entidades Locales, se arbitra un mecanismo similar, que llega a prever la posibilidad de disolución de la Entidad.

De nuevo en este apartado, el Consejo de Estado viene a poner de manifiesto que los procedimientos, plazos y requisitos están muy poco desarrollados en el texto. Se trata de cuestiones altamente sensibles, que afectan al principio de autonomía y que no pueden dejarse al posterior desarrollo mediante simple orden ministerial, como es el caso, por ejemplo, de la composición de la comisión de expertos (antes denominada delegación de expertos en una nueva muestra de la traducción automática).

Pero sin duda, uno de los aspectos más polémicos de la norma y por el que el Consejo de Estado demuestra preocupación es el de la aplicación en este ámbito del procedimiento previsto en el art. $155 \mathrm{CE}$ de la Constitución. A este respecto el Consejo comienza cuestionándose la propia conveniencia de mencionar expresamente este artículo, suponemos que por la carga de amenaza implícita que su mera mención conlleva.

De nuevo respecto de la regulación que hace el anteproyecto, el Consejo de Estado denuncia por enésima vez la falta de precisión tanto del supuesto de hecho que lo origi-

6 T. DE LA QUADRA-SALCEDO JANINI, «La discrecionalidad política del ECOFIN en la aplicación del procedimiento por déficit excesivo. Reflexiones tras la Sentencia del Tribunal de Justicia de 13 de julio de 2004», Revista de estudios políticos, n 126, 2004, págs. 151-176. 
na como de las consecuencias jurídicas que de su aplicación se deriven. En efecto, considera el Consejo que el supuesto de hecho debe ser consistente con el previsto en la Constitución, de modo que «si se va a establecer expresamente que el incumplimiento de una determinada obligación desencadene el mecanismo del artículo 155 de la Constitución, resulta necesario que esa obligación se recoja en la Ley proyectada de forma clara, expresa y terminante». Asimismo, y por idénticos motivos, aprecia que el requerimiento formulado al Presidente de la Comunidad Autónoma incumplidora no puede tener por objeto, según afirmaba el anteproyecto, que lleve a cabo cualesquiera medidas, «sino que su objeto ha de ser, precisamente, el cumplimiento de las obligaciones que han quedado incumplidas (y que han desencadenado la aplicación de este mecanismo extraordinario)».

Por el contrario, se considera acertado que en el anteproyecto no se especifiquen las concretas medidas que pueden adoptarse, pues debe mantenerse el carácter abierto de que le dota la Constitución. Se alude en este sentido a lo sostenido por el propio Consejo de Estado en el Informe sobre las garantías del cumplimiento del Derecho Comunitario, de 15 de diciembre de 2010, respecto de que «la tipología de medidas que pueden ser adoptadas es muy amplia y la opción por unas u otras dependerá de la situación concreta que se trate de solventar». En definitiva, se trata de lograr el cumplimiento forzoso de las obligaciones por parte de la Comunidad Autónoma, por lo que tendrán preferencia aquellas «medidas que no supongan una ejecución subsidiaria o una ejecución por sustitución por parte del Estado, sino, más bien, una intimación o presión para que sea la propia Comunidad Autónoma la que cumpla», y en este sentido se sugieren medidas como la imposición de multas coercitivas a la Comunidad Autónoma incumplidora.

En definitiva, puede decirse que el Consejo a lo largo de todo el Dictamen, aboga por lograr una mayor claridad en la definición tanto de los supuestos de hecho que generan la adopción de las distintas medidas, como de las consecuencias jurídicas que se deriven de su aplicación, al tiempo que denuncia, de manera más o menos velada, algunas posibles vulneraciones del principio de autonomía financiera en que el anteproyecto incurre.

$* * *$

TITLE: Comment on the Report of the Council of State on 1st March 2012, regarding the "preliminary draft of the organic statute (Ley orgánica) on budgetary stability and financial sustainability».

ABSTRACT: The Council of State analyzes the content of the preliminary draft of the organic statute (Ley orgánica) on budgetary stability and financial sustainability; it highlights the fact that the Statute itself is to develop the latest reform of the article 135 in the Spanish constitution. The Council of State places the greatest importance on the recent jurisprudence of the Constitutional Court

RESUMEN: Se trata de un comentario del Dictamen del Consejo de Estado relativo al anteproyecto de Ley Orgánica de Estabilidad Presupuestaria y Sostenibilidad Financiera de las Administraciones Públicas aprobado por el Consejo de Ministros el 13 de febrero. El comentario aborda las principales cuestiones jurídicas tratadas por el citado Dictamen. En él el Consejo de Estado destaca la necesidad de vincular esta norma directamente con la reforma operada en el art. 135 de la Constitución, para lo cual encuentra apoyo en la más reciente doctrina del Tribunal Constitucional

KEY WORDS: Council of State, budgetary stability, financial sustainability, financial autonomy.

Palabras Clave: Dictamen, Consejo de Estado, Estabilidad Presupuestaria, Sostenibilidad Financiera, Autonomía Financiera.

FECHA DE RECEPCIÓN: 22.06.2012. FECHA DE ACEPTACIÓN: 19.07.2012

UNED. Teoría y Realidad Constitucional, núm. 30, 2012, pp. 535-550. 